\title{
A case report of primary cutaneous natural killer/T-cell lymphoma
}

\author{
CHEN TIAN，YONG YU and YIZHUO ZHANG \\ Key Laboratory of Cancer Prevention and Therapy, Tianjin Medical University Cancer Institute \\ and Hospital, National Clinical Research Center for Cancer, Tianjin 300060, P.R. China
}

Received April 1, 2016; Accepted September 9, 2016

DOI: $10.3892 / \mathrm{mco} .2016 .1042$

\begin{abstract}
Primary cutaneous extranodal natural killer/T-cell lymphoma, nasal type (ENKL-NT) is a relatively rare disease associated with aggressive tumor-cell behavior and poor prognosis. Progress in immunohistochemistry has improved the identification of ENKL-NT. The present case study reported on a 64-year-old female patient presenting with several red nodular lesions on the neck developed over four months. Cutaneous biopsy revealed these cells were positive for CD3, CD56, CD5, CD8 and negative for CD2, CD34, CD7, CD20 and Granzyme B. A computed tomography scan and bone marrow biopsy did not show any abnormalities and a diagnosis of primary cutaneous ENKL-NT was made. After treatment with chemotherapy regimens comprising cyclophosphamide, doxorubicin, vincristine and prednisone (CHOP), followed by high-dose treatment with methotrexate, dexamethasone, ifosfamide, etoposide and L-asparaginasum (SMILE), the patient succumbed to the disease. The present study exemplified that immunohistochemical analysis as well as the recognition of atypical lymphoid cells showing angiocentricity is crucial for the correct diagnosis of ENKL-NT. The prognosis of primary cutaneous ENKL-NT remains poor. As the CHOP regimen is not completely effective, high-dose chemotherapy, such as the SMILE regimen, is required.
\end{abstract}

\section{Introduction}

Patients with extranodal natural killer/T-cell lymphoma, nasal type (ENKL-NT), which is prevalent in East Asian countries, usually present with localized extranodal disease (1). The identification of primary cutaneous ENKL-NT has improved with the application of immunohistochemical analysis of markers including CD2, CD3, CD56, T-cell-restricted intracellular

Correspondence to: Dr Yizhuo Zhang or Dr Yong Yu, Key Laboratory of Cancer Prevention and Therapy, Tianjin Medical University Cancer Institute and Hospital, National Clinical Research Center for Cancer, 1 Huanhuxi Road, Hexi, Tianjin 300060, P.R. China

E-mail: yizhuozhang111@163.com

E-mail: yuyongy@163.com

Key words: natural killer/T-cell lymphoma, primary cutaneous, SMILE antigen 1 (TIA-1) and Granzyme B (2). Primary cutaneous ENKL-NT has an aggressive course and a poor outcome despite chemotherapy and radiotherapy (3). The present study reported on an original case with primary cutaneous ENKL-NT.

\section{Case report}

A 64-year-old female patient initially presented with several red nodular lesions on the neck developed over four months. Subsequently, similar multiple erythematous plaques appeared on the patient's face, arms and lower extremities. The lesions were $\sim 4 \times 3 \mathrm{~cm}$ in size, protruding from the surface of the skin. The patient did not have any complaints of pain, while having low-grade fever and weight loss of $5 \mathrm{~kg}$ before hospitalization. The first cutaneous biopsy in December 2015, at an external hospital indicated inflammatory granuloma. Anti-inflammatory treatment did not improve the patient's condition, while the plaques appeared to suppurate. The patient was then admitted to our hospital (Tianjin Medical University Cancer Institute and Hospital, Tianjin, China). Ultrasonic B examination showed lymphadenopathy in the left axilla and bilateral groin. A computed tomography (CT) scan of the chest and abdomen revealed mediastinal invasion and hepatosplenomegaly. The results of a full blood count and liver function tests were within the normal range. Serum lactate dehydrogenase was elevated to 1,148 IU/1 (normal range, 200-460 IU/1). The second cutaneous biopsy performed at our hospital indicated ENKL-NT, as cells were positive for CD3, CD56, CD5, CD8, TIA-1 and negative for CD2, CD34, CD7, CD20 and Granzyme B. Analysis of bone marrow aspirate showed no involvement. The patient was diagnosed with primary cutaneous ENKL-NT and was first treated with cyclophosphamide, doxorubicin, vincristine and prednisone (CHOP regimen) (4), but showed no improvement. Subsequently, a high-dose chemotherapy regimen comprising methotrexate, dexamethasone, ifosfamide, etoposide and L-asparaginasum (SMILE) was used. One month later, the patient succumbed to the disease.

\section{Discussion}

ENKL-NT has a markedly increased prevalence in East Asia, including China. It usually invades the upper aerodigestive tract, including the nasal cavity in $>80 \%$ of affected patients. The skin is the second most frequently involved site (5). 

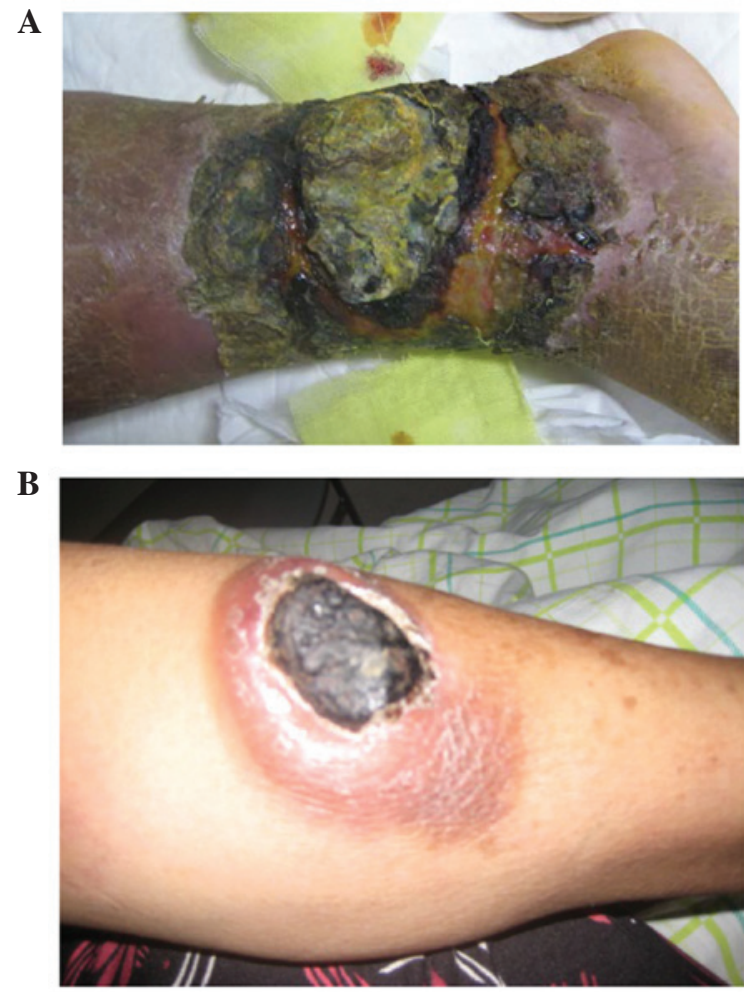

Figure 1. Erythematous plaque lesions on (A) the ankle and (B) left arm. The images were captured in January 2016.

Patients with primary cutaneous ENKL-NT often present with cutaneous nodules or plaques with systemic symptoms including fever, malaise, weight loss and hemophagocytic syndrome. The identification of ENKL-NT has improved with the use of immunohistochemistry, with positivity for markers including CD3, CD5, CD8 and CD56 being specific characteristics. In addition, patients with ENKL-NT are usually positive for Epstein-Barr virus DNA (6). Staging procedures often include clinical examination, complete blood analysis, CT scan of the thorax, abdomen and pelvis, and analysis of bone marrow aspirate.

The median age of patients with primary cutaneous ENKL-NT is 50 years (7). Primary cutaneous ENKL-NT is an aggressive lymphoma type with poor prognosis. According to previous studies, the majority of patients with primary cutaneous ENKL-NT succumbed within a short time $(<1$ year) (8). The patient presented in the current case report succumbed within two months of the diagnosis.

In the clinic, early-stage cases (I and II), in which lesions are confined to a single region, are treated by irradiation therapy, following which the prognosis is rather favorable. However, in cases with multiple cutaneous plaques, ENKL-NT has a highly aggressive course, and patients are treated with multi-agent chemotherapies based on combinations of cyclophosphamide, vincristine and prednisolone. However, several studies have indicated that the efficacy of CHOP against ENKL-NT is inadequate, as disease progression often occurs during $\mathrm{CHOP}$ chemotherapy $(9,10)$. In the present case, as the state of the patient was inferior at first, the CHOP regimen was used. However, disease progression occurred during chemotherapy with CHOP. High-dose chemotherapy is recommended (11).
After treatment using the SMILE regimen, the plaque lesions significantly improved. However, one month later, the patient succumbed due to disease progression. A previous study has reported on the approach of radiotherapy combined with chemotherapy with the complete remission (CR) rate of $29 \%$ (12). The findings of the present and previous studies indicated that the prognosis of patients with ENKL-NT remains poor, irrespective of the treatment modality used.

\section{Acknowledgements}

The present study was supported by grants from the National Natural Science Foundation of China (nos. 31301161, 81670104 and 81270603).

\section{References}

1. Lim ST, Hee SW, Quek R, Lim LC, Yap SP, Loong EL, Sng I, Tan LH, Ang MK, Ngeow J, et al: Comparative analysis of extra-nodal NK/T-cell lymphoma and peripheral T-cell lymphoma: Significant differences in clinical characteristics and prognosis. Eur J Haematol 80: 55-60, 2008.

2. Yeang SH, Chan A, Tan CW, Lim ST and Ng HJ: Incidence and Management of toxicity associated with LAsparaginase in the treatment of ALL and NK/Tcell lymphoma: An Observational Study. Asian Pac J Cancer Prev 17: 3155-3160, 2016.

3. Gupta VG and Gogia A: DDGP versus SMILE in NK/T-cell lymphoma-letter. Clin Cancer Res 22: 4271, 2016.

4. Takahashi T, Kumanomidou S, Takami S, Okada T, Adachi K, Jo Y, Ikejiri F, Onishi C, Kawakami K, Miyake T, et al: A retrospective study of R-CHOP/CHOP therapy-induced nausea and vomiting in non-Hodgkin's lymphoma patients: A comparison of intravenous and oral 5-HT3 receptor antagonists. Int J Hematol 104: 378-383, 2016.

5. Miyagawa F, Iioka H, Fukumoto T, Kobayashi N and Asada H: A case of CD8(+) primary cutaneous peripheral T-cell lymphoma arising from tissue-resident memory T (TRM ) cells in the skin. Br J Dermatol 173: 612-614, 2015.

6. Koizumi K, Fujimoto K, Haseyama Y, Endo T, Nishio M, Yokota K, Itoh T, Sawada K and Koike T: Effective high-dose chemotherapy combined with CD34+-selected peripheral blood stem cell transplantation in a patient with cutaneous involvement of nasal NK/T-cell lymphoma. Eur J Haematol 72: 140-144, 2004.

7. Yoon DH, Kim SJ, Jeong SH, Shin DY, Bae SH, Hong J, Park SK, Yhim HY, Yang DH, Lee H, et al: Phase II trial of concurrent chemoradiotherapy with L-asparaginase and MIDLE chemotherapy for newly diagnosed stage I/II extranodal NK/T-cell lymphoma, nasal type (CISL-1008). Oncotarget: Aug 16, 2016 (Epub ahead of print).

8. Stokkermans-Dubois J, Jouary T, Vergier B, Delaunay MM and Taieb A: A case of primary cutaneous nasal type NK/T-cell lymphoma and review of the literature. Dermatology 213: 345-349, 2006.

9. Matsue K, Fujiwara H and Sandoh E: (18)F-Fluorodeoxyglucose positron emission tomography in primary cutaneous NK/T cell lymphoma, nasal type. Eur J Haematol 82: 493-494, 2009.

10. Wang P, Sun Y, Wang Z and Xu A: Primary cutaneous extranodal NK/T-cell lymphoma, nasal type, in an adolescent. Eur J Dermatol 20: 130-131, 2010.

11. Li X, Cui Y, Sun Z, Zhang L, Li L, Wang X, Wu J, Fu X, Ma W, Zhang $\mathrm{X}$, et al: DDGP versus SMILE in newly diagnosed advanced natural Killer/T cell lymphoma: A randomized controlled, multicenter, open-label study in china. Clin Cancer Res: Apr 8, 2016 (Epub ahead of print).

12. Sitthinamsuwan P, Pongpruttipan T, Chularojmontri L, Pattanaprichakul P, Khuhapinant A and Sukpanichnant S: Extranodal NK/T cell lymphoma, nasal type, presenting with primary cutaneous lesion mimicking granulomatous panniculitis: A case report and review of literature. J Med Assoc Thai 93: 1001-1007, 2010. 